\title{
Using genetic algorithms to improve support vector regression in the analysis of atomic spectra of lubricant oils
}

\author{
Carlos Fernandez-Lozano, Francisco Cedrón, Daniel Rivero and Julian Dorado \\ Information and Communications Technologies Department, University of A Coruña, A Coruña, Spain \\ José Manuel Andrade-Garda \\ Analytical Chemistry Department, University of A Coruña, A Coruña, Spain, and \\ Alejandro Pazos and Marcos Gestal \\ Information and Communications Technologies Department, University of A Coruña, A Coruña, Spain
}

\begin{abstract}
Purpose. - The purpose of this paper is to assess the quality of commercial lubricant oils. A spectroscopic method was used in combination with multivariate regression techniques (ordinary multivariate multiple regression, principal components analysis, partial least squares, and support vector regression (SVR)).

Design/methodology/approach. - The rationale behind the use of SVR was the fuzzy characteristics of the signal and its inherent ability to find nonlinear, global solutions in highly complex dimensional input spaces. Thus, SVR allows extracting useful information from calibration samples that makes it possible to characterize physical-chemical properties of the lubricant oils.

Findings. - A dataset of 42 spectra measured from oil standards was studied to assess the concentration of copper into the oils and, thus, evaluate the wearing of the machinery. It was found that the use of SVR was very advantageous to get a regression model.

Originality/value. - The use of genetic algorithms coupled to SVR was considered in order to reduce the time needed to find the optimal parameters required to get a suitable prediction model.
\end{abstract}

Keywords

Genetic algorithms; Lubricant oils; Support vector regression

\section{Introduction}

The use of lubricant oils whenever moving metallic pieces get in close contact is mandatory to avoid the deterioration of the metal surfaces and to avoid mechanical and thermal stress that may result in structural damage. Lubricant oils create a protective layer over the metallic surfaces so that friction is diminished and premature wearing of the moving parts is prevented, augmenting the useful lifetime of the machinery. Lubricants are complex mixtures of hydrocarbons made up of several mineral and synthetic base oils and additives. Although the use of lubricants reduces friction, this does not prevent the metallic surfaces from releasing metal particles in the oil. Hence, if it were possible to quantify a particular metallic element in the liquid phase of the lubricants it would be possible, in principle, to evaluate the wearing of the machines.

In order to measure the concentration of a given metal (e.g. copper, iron, titanium, etc.) in a lubricating oil a chemical analytical methodology known as electrothermal atomic spectrometry can be employed. Roughly, spectrometry is about the differential reflection and/or absorption of light (radiation). Besides, a sample will respond differently to distinct wavelengths due to the chemical composition of the molecules or atoms that constitute it. The graphical representation of the amount of energy (light or radiation) absorbed (reflected) by the sample as a function of the wavelength of the radiation focused over it is called a spectrum. If only a particular kind of atom is determined, an atomic spectrum is obtained; otherwise a molecular spectrum is measured. 
Establishing a functional numerical relationship between the atomic spectrum of a lubricant oil and the total amount of a metal (which was considered relevant to evaluate machine wearing by technicians) dissolved or suspended in the oil is the first step in assessing the degree of wearing of a machine. The high difficulty in modelling such a relationship makes this problem a perfect candidate to be addressed by means of advanced, multivariate regression techniques. In this paper we compare some popular ones: ordinary multivariate multiple regression (MLR), principal components regression (PCR) and support vector regression (SVR). In this work, SVR seemed particularly suited for this task because of their ability to model non-linear relations and yield global models when complex input information is handled, and also because it is capable of dealing with high-dimensional input spaces.

In this paper a SVR model is proposed to measure the content of a metal (copper) in 42 standard oils whose atomic spectra were registered following a dedicated analytical protocol (Carballo et al., 2013). In addition, the use of genetic algorithms (GA) to automatically and objectively optimize the SVR parameters required to get a suitable predictive model is presented as well.

The current paper is organized as follows: the theoretical background section describes the particular methods used in this work; the materials and methods section describes the methodology; the results and discussion section includes a comparison of the proposed algorithm with some state-of-the-art regression algorithms; finally, the conclusions are presented.

Hence, the aim of this paper is to evaluate the capability of a hybrid approach using GA to optimize a SVR model.

\section{Theoretical background}

In empirical data modelling a process of induction is used to build up a model of the system. This is expected to yield responses of the system that have not yet been observed. It is worth recalling here that experimental (observed) data are finite and will form a sparse distribution in the input space according to the high-dimensional nature of the non-uniform sampling of the data. Therefore, this might compromise the correct optimization of the model and, so, GA appears as a superb tool to ameliorate the experimental workload in the laboratories.

Here, a hybridization among GA (Holland, 1975) and support vector machines (SVM) (Vapnik, 1979) is presented. More specifically SVM for function estimation, SVR (Smola and Schölkopf, 2004), are a non-linear generalization of the original algorithm. In this section, the main techniques used are introduced and explained briefly. This approach may constitute an alternative to other three well-known ways of controlling the performance of SVR; namely, the loss function, the kernel and the so-called additional capacity model (Brereton and Lloyd, 2010; Gunn, 1998).

\subsection{SVM}

Vapnik introduced SVMs in the late 1970s on the foundation of statistical learning theory (Vapnik, 1979). Its basic implementation dealt with two-class problems in which data were separated by a hyperplane defined by a number of support vectors. The hyperplane separates the "positive" from the "negative" examples, so that the distance between the boundary and the nearest data point in each class is maximal; these data points are used to define the margins, and are called support vectors (Burges, 1998). SVMs have proved to be exceptionally efficient in classification problems of higher dimensionality (Moulin et al., 2004; Chapelle et al., 1999; Fernandez-Lozano et al., 2013a), because of their ability to generalize in high-dimensional spaces. SVM uses different non-linear kernel functions, like polynomial, sigmoid and radial basis function, where the non-linear SVM maps the training samples from the input spaces into a higher-dimensional feature space via a mapping function (Burges, 1998). The key idea behind SVMs is to use a non-linear mapping function that transforms the input data space in such a way as to render a problem linearly separable.

The SVM formulation embodies the structural risk minimisation (SRM) principle which has been shown to be superior than the traditional empirical risk minimisation (ERM) principle (employed, e.g. for neural networks) (Gunn et al., 1997). Following, SVM generalizes better than conventional neural networks, which is the final goal in statistical learning, because SRM minimizes an upper bound on the expected risk whilst ERM minimizes the error on the training data.

In the beginning, SVMs were developed for classification problem solving but they have been extended to the domain of regression and estimation problems. A SVM for solving regression problems is called SVR. In addition to the method proposed by Vapnik (1979) an alternative loss function is proposed (Smola and Schölkopf, 2004) to include a distance measure. This loss function could produce no 
sparseness in the support vectors (i.e. least squares error, Laplacian or Huber) or sparseness with an approximation to Huber's loss function. Instead of minimizing the observed training error, SVR attempts to minimize the generalization error bound to achieve improved general performance.

As we previously noted, there are three general methods for controlling the performance of the SVR model: the loss function, the kernel and the additional capacity control (Brereton and Lloyd, 2010; Gunn, 1998). These aspects present different parameters that should be established with correct values (usually from an expert in the field of study) to provide the desired results. At this point, GA (Goldberg, 1989; Holland, 1975) can provide excellent results in order to find the correct parameters by means of a "guided" search. This kind of algorithms can also perform a previous phase of feature selection to determine how variables are more important or provides more information among the whole dataset (Hu et al., 2014) and improve in that way the regression results.

SVRs have been compared against other regression models on benchmark time series prediction tests (Mukherjee et al., 1997), the Boston housing problem and the PET operator inversion problem, with either similar or significantly best performance (Burges, 1998) than other usual methodologies. However, to the best of our knowledge there are not comparisons with common regression methods in analytical laboratory environments.

\section{$2.2 G A$}

GAs are exhaustive and intensive search techniques inspired by Darwinian evolution and developed by Holland in the 1970s (Holland, 1975). In a GA, an initial population of individuals, each one a possible solution to the problem, is evolved by means of genetic operators: selection, crossover and mutation (see Figure 1 for a general scheme of their functioning). Prior to the implementation of the GAbased optimization process, it is necessary to determine how to represent the solutions (encoding, usually by means of binary or real values) and how to measure how good is the proposed solution (fitness function). Thus, the goodness of every individual solution is measured by means of a fitness function, indicating a real value representing this value. As in nature, the selection operator ensures the survival of the fittest, while the crossover represents the mating between individuals, and the mutation operator introduces random modifications.

There are several options for every of those algorithms. Selection algorithms pick up two individuals from the population, usually related with their fitness value. Most used selection algorithms are the roulette wheel selection (probability of selection for one individual is proportional to the individual fitness) or the tournament selection (it performs "tournaments" among a few individuals chosen at random from the population and the finally selected individual will be the one with the best fitness among the selected for the "tournaments". This algorithm allows to change the selection pressure by changing the tournament size.

Among the crossover algorithms the most used are the one-point crossover, two-point crossover or uniform crossover. All of them merge information from two parents to generate the offspring. Crossover rates are usually around 95 per cent.

Mutation algorithm is the responsible to keep the genetic diversity in a population introducing new values for the genes as generations advance, like the biological mutation. Most used mutation algorithms are the bit inversion (that changes the value of a bit from 0 to 1 or vice versa) or the replacement/swapping (that interchanges the values of two genes within the genetic individual). Mutation rates are usually very low in comparison with crossover rates, about 1-2 per cent.

At the end of every generation GA offspring should be inserted in the population. Another time, there are several options, with or without the use of temporal populations, but in all of them some of the actual individuals (a random individual, the parents, the worst individuals, etc.) in the population, are replaced with the generated offspring by means of the crossover and mutation algorithms. 


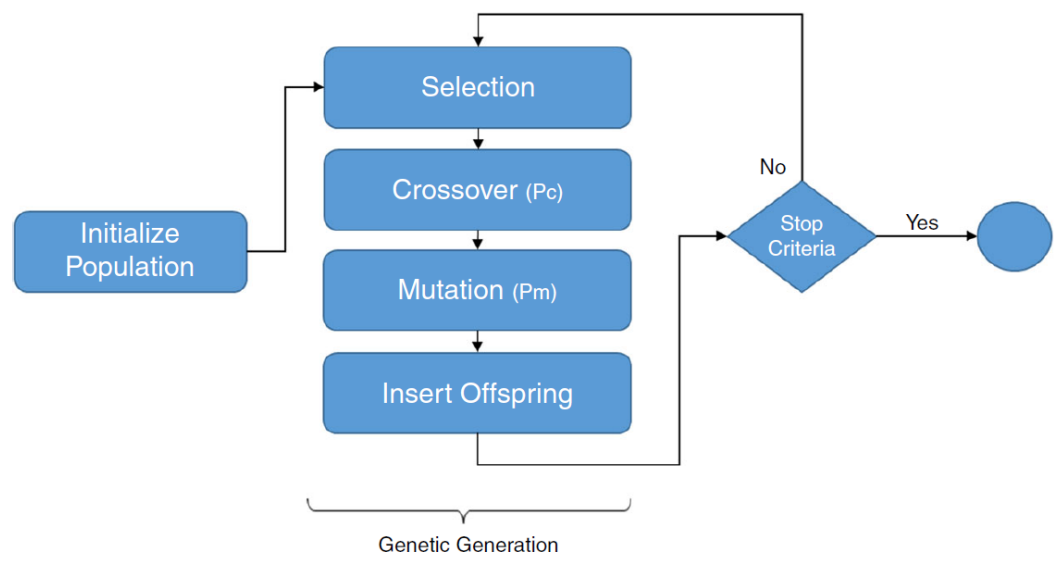

Figure 1. Genetic algorithm: general schema

GA possesses effective exploration and exploitation capabilities to explore the search space in parallel, exploiting the information about the quality of the individuals evaluated so far (Goldberg, 1989). A chromosome in the population represents a candidate solution to the particular problem. It is commonly represented by a string of binary numbers where each set of binary values represents a feature measure (Goldberg, 1990), although that real representations are also available.

The power of GA-based approaches lies in the massively parallel exploration of the input space. It is mainly due to the fact that several possible solutions can occur for a particular problem, each exploring an area of the input space. The set of solutions (genetic population) is randomly initialized.

By means of the crossover operator, GA combines the features of the parents to produce new and usually better solutions, if the parent's best characteristics are preserved. The selection algorithms will be responsible for choosing the best individuals to reproduce (the selection of the individual is related to its fitness value). By using the mutation operator, new information is introduced in the population in order to explore new and promising areas of the search space (the value of one of its genes varies randomly). Mutation is often used together with the crossover operator. Another strategy known as elitism, which is a variant of the general process of constructing a new population, is used to allow best organisms from the current generation to be carried out to the next one, unaltered. At the end of the process, it is expected that the population of solutions converge to the global optimum guided by the fitness function. Thus, GA refine solutions which will gradually be closer to obtaining a good overall solution of the problem.

The crossover operator combines the features of two parents to form two offsprings; it means that this operator is a method for sharing information. In binary-coded GA the classical crossover operator is the simple crossover, but there are also more powerful operators such as the multi-point and uniform crossover (Picado et al., 2009). There are other approaches that use several populations, specific crossover or mutation algorithms and so on with the aim to improve some of the GAs characteristics.

This is mainly an iterative process and therefore, it should be necessary to specify a criterion that allows establishing when this process is complete. There are several different approaches but the most common are that the fittest individual is good enough for solving this problem, more than the 95 per cent of the population has the same value (the population has converged), the difference of the best individual and the rest of the population is reduced or the maximum number of generations have been reached.

The most important aspect in the GAs is the aforementioned fitness function. This function measures the relative importance of a specific solution or individual, that is, how good is the individual providing a final solution to the problem. At this step is common to use hybrid approaches or getting that measure by means of the use of techniques that usually offers a good approximation to solve problems in the field of study. It can include the use of artificial neural networks, SVM as well as other classifiers. 


\section{Materials and methods}

As mentioned above, to quantify copper in lubricant oils it is necessary to register the atomic spectrum of the samples. Unfortunately, when measuring such complex samples strong interferences on the required signal appear due to the sample concomitants. Hence, the information gathered from the atomic analyses has usually some fuzzy characteristics (random noise, slight signal displacements, chemical interferences, etc.), which make it not suitable to get a direct, simple solution. Therefore, it is of most importance to develop, from the recorded spectroscopic data, regression models that allow an indirect quantification of the amount of copper in the oils and, thus, indirectly evaluate the wearing of a machine.

A calibration process with synthetic oils developed into the laboratory was undergone. The calibration process used 34 lubricants, whereas the validation group consisted of eight oils.

The hybrid approach proposed in this work makes use of the LibSVM (Chang and Lin, 2011) nu-SVR implementation within the fitness function to measure how good is each one of the solutions provided by the GA, developed with the GAlib library (Wall, 1996). In our method, both GA and SVR are combined in order to avoid the limitations derived from the need to optimize manually the SVR parameters. In this particular implementation, LibSVM uses a parameter, called nu, to control the number of support vectors used within the process. LibSVM was coupled to both a standard GA developed previously for classification (Fernandez-Lozano et al., 2013b) and a multimodal GA (Gómez-Carracedo et al., 2007a, b). A regularization parameter, $\mathrm{C}$, controls the trade-off between the classifier complexity and the number of non-separable points. This common approach was preferred here instead of other new ones (e.g. those based on firefly and memetic algorithms (Hu et al., 2013) or particle swarm optimization (Alwee et al., 2013)).

The LibSVM kernel implementation required three additional parameters to be optimized: the degree of the polynomial (whether a polynomial kernel is used), a $\gamma$ factor (when the polynomial, radial basis function or sigmoid kernels are considered), and a c coefficient (in the polynomial and sigmoid kernels).

Due to the high number of individual parameters and the wide range of possible values for each, it is not possible to test all parameter combinations to search for the best model. Following, this work proposes the use of GA to address this problem. GA is aimed at finding the best combination of the SVR parameters to generate the most suitable regression model. Each individual in the genetic population is described using binary encoding and with non-fixed length. This means that, in the same population, individuals that represent different kernel functions will coexist and, according with their particular kernels, the number of genes will be different (see Table I for some examples).

\begin{tabular}{lc} 
Table I. GA individual lengths & \\
\hline Kernel function & Individual length \\
\hline Linear & 44 \\
Radial basis & 61 \\
Sigmoid & 82 \\
Polynomial & 103 \\
\hline
\end{tabular}

This option was initially considered the best because it avoids an overload of the number of parameters that GA should optimize so the computational requirements and time needed to get the results were reduced without penalize too much the quality of the solutions provided. Once this approach seems valid to provide good results, another option that should be studied more in depth is the inclusion of the kernel function as other of the parameters optimized within the GA.

The genetic population uses a binary codification in order to represent different kernel parameters, which can be either integer or a float number. The latter is represented in binary-code using three different parts and with different precision; sign, exponent and mantissa. In Table II the different parameters optimized by the GA-SVR approach are shown. The final values were extracted among the most used values in the related works and after several tests that determined the most promising ranges. 
Table II. List of parameter setting

\begin{tabular}{llcc}
\hline Parameter & Format & Precision/decimal places & Range \\
\hline & & & \\
$\mathrm{Nu}$ & Float & 7 & {$[0,1]$} \\
$\mathrm{C}$ & Float & 3 & {$[0,1023]$} \\
Degree & Integer & - & {$[0,31]$} \\
$\gamma$ & Float & 5 & {$[0,1]$} \\
$\mathrm{c}$ & Float & 3 & {$[-1023,1023]$}
\end{tabular}

\section{Results and discussion}

The overall set of samples was composed of 42 synthetic lubricant oil standards separated in a calibration group (34 standards) and a validation group (eight ones, not used at all for calibration purposes). The experimental measurement of each atomic spectrum (Bordé, 1989; Corney, 2006) yielded 216 measures of the absorbance signal (at the same wavelength), each at a different time (from 0 to $2.5 \mathrm{~s}$ ) (Figure 2). Data are normalized between values $[0,1]$ in pre-process step.

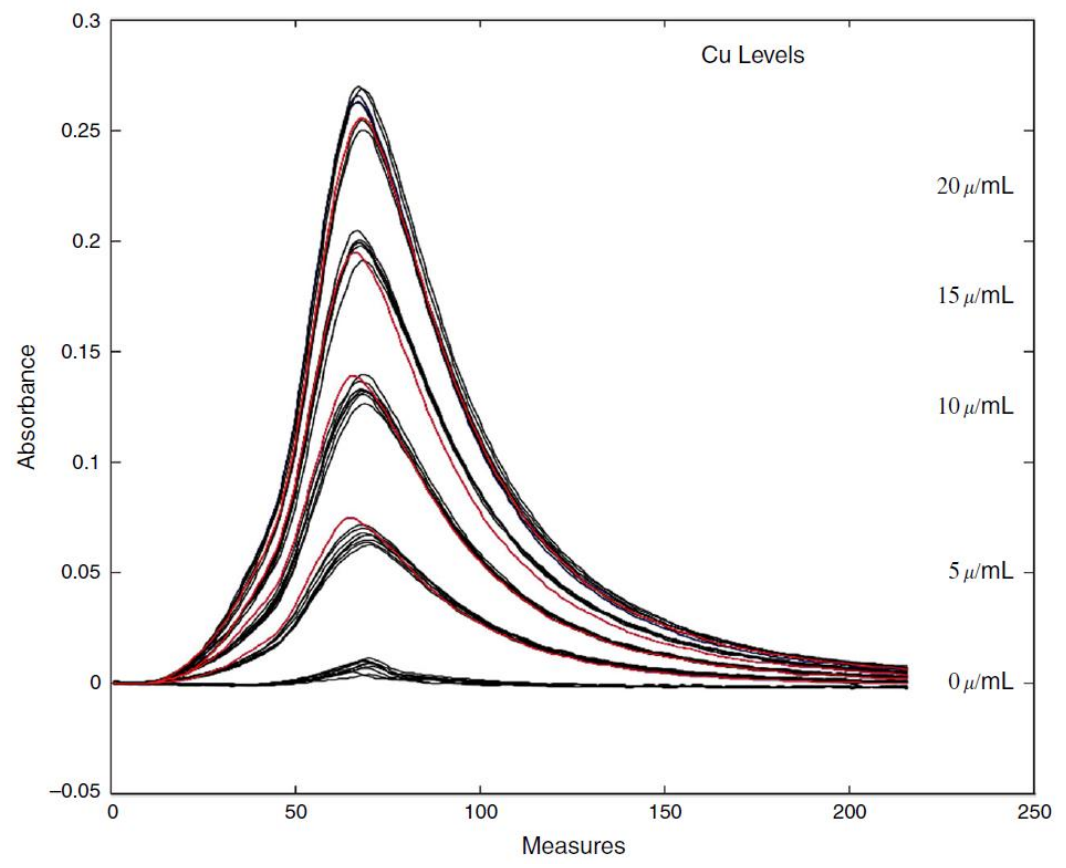

Figure 2. Atomic spectra of the oils

In this case, the absorvance signal will show us the level of presence of copper in the sample. This value will give us information about of the probability of fail of one engine, for example, so it will be higher in engines with more friction and/or wear.

These are an own-purpose samples build from synthetic lubricant oil standards used as input for an atomic spectra tool, but there is wide number of works related with the assessment of the quality of commercial lubricant oils using different approaches to get the indicators: optical fibre spectroscopy (Mignani et al., 2009), X-ray (Pouzar et al., 2001) or infrared spectroscopy (Caneca et al., 2006). These woks offers different approaches to measure the quality of the oil and, in a future could be used to test the proposed method with different input data in order to check the goodness of the proposed technique.

The parameters domains for the SVR optimization were set as indicated in Table III. They were adjusted initially according to a previous search of the most common values in literature. 
Table III. GA parameters domain

\begin{tabular}{ll}
\hline Item & Domain \\
\hline Population size & From 40 to 100 \\
Crossover probability & From 90 to $99 \%$ \\
Mutation probability & From 0 to 5\% \\
Crossover operators & One-point crossover, two-point crossover, uniform \\
Selection function & Roulette, deterministic and probabilistic tournament (tournament size, 2-4) \\
Mutation function & Bit inversion, bit swapping \\
Replacement algorithm & Parents, worst individuals \\
&
\end{tabular}

A series of trials were carried out to optimize the values of the parameters. The most satisfactory combination included a population size of 60 individuals, with a tournament selection function, bit inversion mutation with probability 0.03 , and uniform crossover operation with probability 0.94 . The fitness function to guide the search can be expressed as follows:

$$
\text { Fitness }=\left(1-R^{2}\right) . M S E
$$

where MSE is the mean square error and R2 is the coefficient of determination that measures how close the data are to the fitted regression line. The best results are shown in Table IV for polynomial and sigmoid kernels, differentiating between the calibration and the validation data. We included also results for a simple linear regression implementation in WEKA (Hall et al., 2009). Ferre-Baldrich and BoqueMarti (2013) applied a PCR and MLR approaches with this dataset and are included as a baseline for comparison purposes with our approach.

As results of Table IV showed, we achieved the better results in validation with the polynomial and the sigmoid kernels.

Table IV. Results

\begin{tabular}{lcccccccc}
\hline & & Linear & Polynomial & Sigmoid & RBF & PCR & MLR & SLR \\
\hline Calibration & MSEC & 0.043 & 0.097 & 0.455 & 0.034 & 0.137 & 0.902 & 0.067 \\
Validation & MSEP & 0.217 & 0.205 & 0.199 & 0.238 & 0.216 & 0.562 & 0.421 \\
& & & & & & & & \\
\hline
\end{tabular}

\section{Conclusions}

In this work a method to relate the atomic spectra of a lubricant oil and its content in copper was developed. It was found that the use of SVR was very advantageous to get a regression model because it can perform both linear and non-linear regression very accurately and its solution is global (Thissen et al., 2004). The proposed method is based on a hybrid approach that combines GA and SVR to get the fitness values. To avoid the high number of valid parameter combinations and the excessive time if manual trials are done, GA will guide the SVR process. Several studies were performed in order to measure the behaviour of the system and to achieve the most adequate model to solve the regression problem.

With the aim of demonstrating the effectiveness of the proposed hybrid model a dataset of 42 spectra measured from oil standards was studied to assess the concentration of copper into the oils and, thus, evaluate the wearing of the machinery. According with our results, we can come to the conclusion that our approach improves the results obtained by other state-of-the-art regression models in both calibration and validation.

In this first preliminary approach only one complex fitness function was tested to evaluate whether the proposed methodology worked correctly with the particular dataset considered. Despite the results were quite good it was studied whether the use of other fitness functions might improve the final results. It will be also interesting in future work to perform some experiments following a feature selection approach in order to select from the original 216 measures of the absorbance signal (at the same wavelength) which 
are the most informative or even to separate measures by collection time in order to evaluate the real influence of the time in the concentration of copper.

\section{References}

Alwee, R., Hj Shamsuddin, S.M. and Sallehuddin, R. (2013), "Hybrid support vector regression and autoregressive integrated moving average models improved by particle swarm optimization for property crime rates forecasting with economic indicators", The Scientific World Journal, Vol. 2013, Article ID 951475, pp. 1-11, available at: http://dx.doi.org/10.1155/2013/951475

Bordé, C.J. (1989), “Atomic interferometry with internal state labelling”, Physics Letters A, Vol. 140 Nos 1-2, pp. 10-12. doi: 10.1016/0375-9601(89)90537-9.

Brereton, R.G. and Lloyd, G.R. (2010), "Support vector machines for classification and regression", Analyst, Vol. 135 No. 2, pp. 230-267. doi: 10.1039/b918972f.

Burges, C.J.C. (1998), "A tutorial on support vector machines for pattern recognition”, Data Mining and Knowledge Discovery, Vol. 2 No. 2, pp. 121-167. doi: 10.1023/A:1009715923555.

Caneca, A.R., Pimentel, M.F., Galvão, R.K.H., Da Matta, C.E., Carvalho, D.E., Raimundo, F.R., Pasquini, I.M.C. and Rohwedder, J.J. (2006), "Assessment of infrared spectroscopy and multivariate techniques for monitoring the service condition of diesel-engine lubricating oils", Talanta, Vol. 70 No. 2, pp. 344-352. doi: 10.1016/j.talanta.2006.02.054.

Carballo, S., Terán, J., Soto, R.M., Carlosena, A., Andrade, J.M. and Prada, D. (2013), "Green approaches to determine metals in lubricating oils by electrothermal atomic absorption spectrometry (ETAAS)", Microchemical Journal, Vol. 108, pp. 74-80. doi: 10.1016/j.microc.2013.01.002, available at: www.sciencedirect.com/science/article/pii/S0026265X13000076

Chang, C.C. and Lin, C.J. (2011), "LIBSVM: a library for support vector machines", ACM Transactions on Intelligent Systems and Technology, Vol. 2 No. 3, pp. 1-27.

Chapelle, O., Haffner, P. and Vapnik, V.N. (1999), "Support vector machines for histogram-based image classification", IEEE Transactions on Neural Networks, Vol. 10 No. 5, pp. 1055-1064.

Corney, A. (2006), Atomic and Laser Spectroscopy, Clarendon Press, Oxford.

Fernandez-Lozano, C., Seoane, J.A., Gestal Pose, M., Gaunt, T.R. and Campbell, C. (2013a), "Texture classification using kernel-based techniques", in Ignacio Rojas, G.J. and Cabestany, J. (Ed.), International Work Conference on Artificial Neural Network, Springer Heidelberg, Puerto de la Cruz, pp. 427-434.

Fernandez-Lozano, C., Canto, C., Gestal, M., Andrade-Garda, J.M., Rabuñal, J.R., Dorado, J. and Pazos, A. (2013b), "Hybrid model based on genetic algorithms and SVM applied to variable selection within fruit juice classification", The Scientific World Journal, Vol. 2013, Article ID 982438, pp. 1-13, available at: http://dx.doi.org/10.1155/2013/982438

Ferre-Baldrich, J. and Boque-Marti, R. (2013), "Ordinary multiple linear regression and principal components regression", in Andrade-Garda, J.M. (Ed.), Basic Chemometric Techniques in Atomic Spectroscopy, Vol. 2, Chapter 4, The Royal Society of Chemistry, Cambridge, pp. 256-279.

Goldberg, D. (1989), Genetic Algorithms in Search, Optimization, and Machine Learning, Addison-Wesley Professional, Boston, MA.

Goldberg, D.E. (1990), "Real-coded genetic algorithms, virtual alphabets, and blocking", Complex Systems, Vol. 5, pp. 139-167.

Gómez-Carracedo, M.P., Gestal, M., Dorado, J. and Andrade, J.M. (2007a), "Chemically driven variable selection by focused multimodal genetic algorithms in mid-IR spectra", Analytical and Bioanalytical Chemistry, Vol. 389 No. 7, pp. 2331-2342. doi: 10.1007/s00216-007-1608-1.

Gómez-Carracedo, M.P., Gestal, M., Dorado, J. and Andrade, J.M. (2007b), "Linking chemical knowledge and genetic algorithms using two populations and focused multimodal search", Chemometrics and Intelligent Laboratory Systems, Vol. 87 No. 2, pp. 173-184. doi: 10.1016/j.chemolab.2006.12.002.

Gunn, S., Brown, M. and Bossley, K. (1997), "Network performance assessment for neurofuzzy data modelling", in Liu, X., Cohen, P. and Berthold, M. (Eds), Advances in Intelligent Data Analysis Reasoning about Data, Springer Berlin Heidelberg, Berlin, pp. 313-323.

Gunn, S.R. (1998), "Support vector machines for classification and regression”, ISIS Technical Report No. 14, Southampton.

Hall, M., Frank, E., Holmes, G., Pfahringer, B., Reutemann, P. and Witten, I.A. (2009), "The WEKA data mining software: an update", SIGKDD Explorations, Vol. 11 No. 1, pp. 10-18.

Holland, J.H. (1975), Adaptation in Natural and Artificial Systems: An Introductory Analysis with Applications to Biology, Control, and Artificial Intelligence, University of Michigan Press, Cambridge, MA.

Hu, R., Jiang, J., Liu, G. and Wang, L. (2014), "Efficient resources provisioning based on load forecasting in cloud", The Scientific World Journal, Vol. 2014, Article ID 321231, pp. 1-12, available at: http://dx.doi.org/10.1155/2014/321231

Hu, Z., Bao, Y. and Xiong, T. (2013), "Electricity load forecasting using support vector regression with memetic algorithms", The Scientific World Journal, Vol. 2013, Article ID 292575, pp. 1-10, available at: http://dx.doi.org/10.1155/2013/292575 
Mignani, A.G., Ciaccheri, L., Díaz-Herrera, N., Mencaglia, A.A., Ottevaere, H., Thienpont, H., Francalanci, S., Paccagnini, A. and Pavone, F.S. (2009), "Optical fiber spectroscopy for measuring quality indicators of lubricant oils”, Measurement Science and Technology, Vol. 20 No. 3, p. 034011.

Moulin, L.S., Alves da Silva, A.P., El-Sharkawi, M.A. and Marks, R.J. II (2004), "Support vector machines for transient stability analysis of large-scale power systems", IEEE Transactions on Power Systems, Vol. 19 No. 2, pp. 818-825. doi: 10.1109/TPWRS.2004.826018.

Mukherjee, S., Osuna, E. and Girosi, F. (1997), "Nonlinear prediction of chaotic time series using a support vector machine", in Principe, J., Gile, L., Morgan, N. and Wilson, E. (Eds), Neural Networks for Signal Processing VII - Proceedings of the 1997 IEEE Workshop, IEEE, New York, NY, pp. 511-520.

Picado, H., Gestal, M., Lau, N., Reis, L.P. and Tomé, A.M. (2009), "Automatic generation of biped walk behavior using genetic algorithms”, Vol. 5517, pp. 805-812. doi: 10.1007/978-3-642-02478-8_101.

Pouzar, M., Černohorský, T. and Krejčová, A. (2001), "Determination of metals in lubricating oils by X-ray fluorescence spectrometry”, Talanta, Vol. 54 No. 5, pp. 829-835. doi: 10.1016/S0039-9140(01)00338-1.

Smola, A.J. and Schölkopf, B. (2004), "A tutorial on support vector regression", Statistics and Computing, Vol. 14 No. 3, pp. 199-222. doi: 10.1023/B:STCO.0000035301.49549.88.

Thissen, U., Pepers, M., Üstün, B., Melssen, W.J. and Buydens, L.M.C. (2004), "Comparing support vector machines to PLS for spectral regression applications", Chemometrics and Intelligent Laboratory Systems, Vol. 73 No. 2, pp. 169-179. doi: 10.1016/j.chemolab.2004.01.002.

Vapnik, V.N. (1979), Estimation of Dependences Based on Empirical Data [in Russian], Nauka, English translation Springer Verlang, 1982, Moscow.

Wall, M. (1996), "GAlib: a C++ library of genetic algorithm components", Mechanical Engineering Department, Massachusetts Institute of Technology, MA. 